\title{
Magnon scattering and tunneling through localized states in magnetic tunnel junctions
}

\author{
J. Wingbermühle, S. Stein, and H. Kohlstedt ${ }^{\mathrm{a})}$ \\ Institut für Festkörperforschung, Forschungszentrum Jülich, 52425 Jülich, Germany
}

(Received 30 April 2002; accepted 4 September 2002)

\begin{abstract}
Spin-dependent tunnel junctions with $\mathrm{Co}_{75} \mathrm{Fe}_{25}$ electrodes and a $\mathrm{Fe}_{50} \mathrm{Mn}_{50}$ pinning layer were fabricated with tunneling magnetoresistance of about $20 \%$ at room temperature. The $\mathrm{Al}_{2} \mathrm{O}_{3}$ barriers were formed by ultra-violet light assisted oxidation. The resistance $\times$ area $(R \times A)$ product was about $375 \mathrm{k} \Omega \mu \mathrm{m}^{2}$ in the parallel alignment of the magnetizations. The effect of barrier impurities has been investigated via tunneling conductance as a function of temperature and bias voltage for as-deposited and annealed samples. The spin wave parameter $\alpha$ was determined to be 3.06 $\times 10^{-5}$ and $2.03 \times 10^{-5} \mathrm{~K}^{-3 / 2}$ before and after annealing, respectively. The improved barrier properties after annealing are explained by inelastic hopping via several localized states and reduced magnon scattering. We propose a qualitative model of the barrier homogenization during annealing which supports former Rutherford backscattering and x-ray photoelectron spectroscopy studies. (C) 2002 American Institute of Physics. [DOI: 10.1063/1.1516844]
\end{abstract}

\section{INTRODUCTION}

The tunneling of electrons via localized states is an important process in relation to many different questions in the framework of electronic transport in solids and devices. This includes, for example, transport in amorphous semiconductors (variable range hopping/Mott hopping), resonant tunneling through tunnel barriers, as well as electron transport through thin dielectric layers. ${ }^{1-3}$ Roughly speaking the two extreme cases of $t \gg l_{\text {in }}$ and $t<l_{\text {in }}$ are quite well understood, where $t$ is the transport length and $l_{\text {in }}$ the characteristic inelastic scattering length. The first one $\left(t \gg l_{\text {in }}\right)$ is described by Mott's theory ${ }^{1}$ of current transport in disordered solids and the second $\left(t<l_{\text {in }}\right)$ by direct elastic tunneling. ${ }^{4}$

Nonetheless the understanding of the intermediate regime $\left(t \approx l_{\text {in }}\right)^{5}$ is important for many device applications. In the case of tunnel junctions trap scattering alters the temperature dependence of the $R \times A$ product in comparison to ideal, defect-free barriers. Traps also lead to undesired lowfrequency excess noise as shown by Rogers and Buhrman in small superconducting tunnel junctions. ${ }^{6}$ So-called Lorentz spectra (as a result of telegraph noise) have been attributed to the capture and emission of electrons in individual traps located inside the barrier or at the barrier interfaces in various barrier materials such as, e.g., $\mathrm{Nb}_{2} \mathrm{O}_{5},{ }^{6} \mathrm{SiO}_{2},{ }^{7,8} \mathrm{In}_{2} \mathrm{O}_{3},{ }^{9,10}$ $\mathrm{MgO},{ }^{11} \mathrm{Al}_{2} \mathrm{O}_{3},{ }^{12}$ and amorphous-Si $(\alpha-\mathrm{Si}){ }^{5,13-16}$ It is known that ionic configurational tunneling of oxygen vacancies may cause such telegraph noise. ${ }^{7}$

Recently high-quality (metallic) ferromagnetic FMI-FM tunnel junctions (here FM is the ferromagnet and I the few $\AA$ thin tunnel barrier) have been developed with potential applications as field sensors or in nonvolatile memories. ${ }^{17-20}$ The function of a magnetic tunnel junction is based on spin-polarized tunneling. Due to the uneven spin distribution of conduction electrons at the Fermi level, the

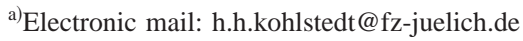

resistance depends on the relative magnetization of the ferromagnetic films. In the simplest theoretical approach, the Jullière model, ${ }^{21}$ both spin channels (spin-up and spin-down) are considered to be independent and that tunneling is an elastic process.

In real tunnel barriers, defects in the form of impurities or oxygen vacancies are present and can lead to considerable deviation in the electronic transport with respect to Jullière's model. ${ }^{22,23}$ The reason, for example, is that traps are coupled to the lattice vibrations and scatter events can lead to spin flipping. Therefore tunneling via traps is not necessarily accompanied by spin conservation. A second source for spin flipping is caused by magnons at the electrode-barrier interfaces. $^{24}$ The generation and annihilation of magnons is accompanied by a spin-flip process. Tunneling via traps as well as magnon excitations may lead to a decrease of the optimal tunneling magnetoresistance (TMR), as predicted by the Jullière model. Therefore the understanding of the barrier and interfaces is essential for fabricating tunnel junctions with high TMR values. In general the current transport in magnetic tunnel junctions is a complex superposition of different conductivities. As mentioned above, the spin-flip processes have magnetic (magnons) or structural (impurities) origins. In this article we present results that distinguish between spin flipping caused by magnons and spin-independent impurity scattering.

\section{EXPERIMENT}

We studied the spin-dependent tunneling through $\mathrm{Al}_{2} \mathrm{O}_{3}$ prepared by UV-light assisted oxidization of magnetic tunnel junctions. ${ }^{25}$ The complete layer structure was $\mathrm{Si}$ substrate $/ \mathrm{Ta}(5 \mathrm{~nm}) / \mathrm{Ni}_{81} \mathrm{Fe}_{19}(3 \mathrm{~nm}) / \mathrm{Cu}(20 \mathrm{~nm}) / \mathrm{NiFe}(3 \mathrm{~nm}) /$ $\mathrm{Fe}_{50} \mathrm{Mn}_{50}(20 \mathrm{~nm}) / \mathrm{Co}_{75} \mathrm{Fe}_{25}(3 \mathrm{~nm}) / \mathrm{Al}_{2} \mathrm{O}_{3}(1.3 \mathrm{~nm} \mathrm{Al}$ plus oxidation $) / \mathrm{Co}_{75} \mathrm{Fe}_{25}(3 \mathrm{~nm}) / \mathrm{NiFe}(20 \mathrm{~nm}) / \mathrm{Au}(20 \mathrm{~nm})$ and was similar to the layer sequence used by Han et l. $^{26}$ The oxidation was done at an $\mathrm{O}_{2}$ partial pressure of 5 mbar for $t$ 


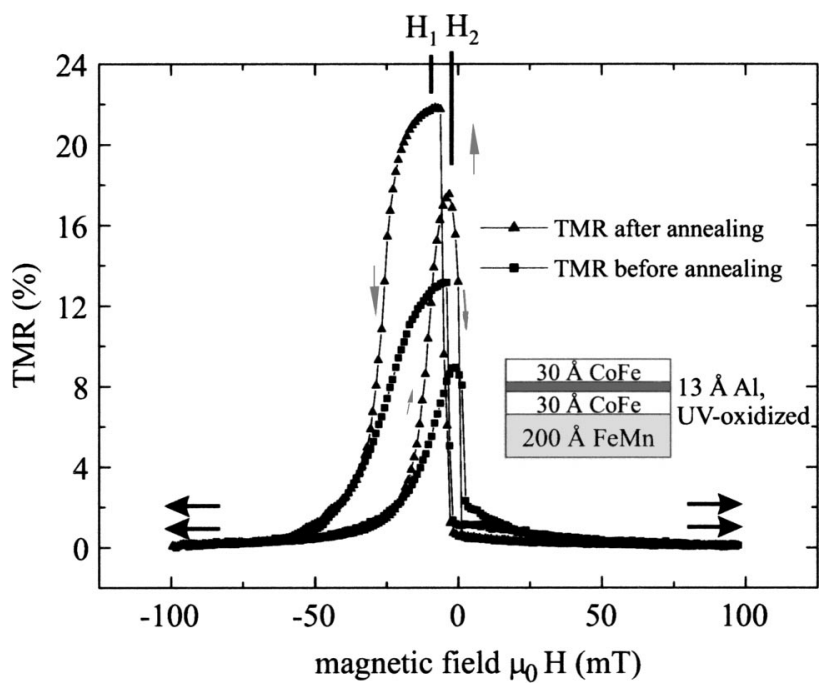

FIG. 1. TMR curve for the as-deposited and annealed samples. The inset shows the schematic cross-sectional view of the tunnel junction including the exchange-bias layer.

$=60 \mathrm{~min}$. The $\mathrm{Ta}$ and first NiFe were used as a seed and a texture layer, respectively, for achieving the (111) direction of the $\mathrm{Fe}_{50} \mathrm{Mn}_{50}$ layer which is necessary for antiferromagnetism and pinning of the adjacent $\mathrm{Co}_{75} \mathrm{Fe}_{25}$ film (the socalled "exchange-bias" effect). The structure was deposited without a magnetic bias field. Junctions with dimensions down to $1 \times 5 \mu \mathrm{m}^{2}$ were patterned by a self-aligned microfabrication process using standard optical lithography and ion-beam etching.

For the resistance and magnetoresistance measurements we used the conventional four-point geometry. The magnetic field was generated by a water-cooled electromagnet and was simultaneously recorded by a Hall probe. The maximal field strength is $\pm 600 \mathrm{mT}$. Using a lock-in amplifier technique the dynamic conductance $d I / d V$ vs $V$ has been measured in the temperature range of 15 to $300 \mathrm{~K}$. All measurements were done in an Oxford cryostat equipped with a controller to stabilize the temperature in a range of about $0.1 \mathrm{~K}$. The electrical characterization was performed before and after annealing. The annealing procedure was done at $200^{\circ} \mathrm{C}$ and then cooled down to room temperature in a magnetic field of $13 \mathrm{mT}$.

\section{RESULTS AND DISCUSSION}

Figure 1 shows the TMR before (solid squares) and after annealing (solid triangles) at room temperature. The gray arrows represent the cycling direction and the black arrows indicate the alignment of the magnetization of the layers. After annealing the TMR increased from $17.5 \%$ to $21.8 \%$. The reason for this is the homogenization and redistribution of the oxygen. Using Rutherford backscattering spectrometry $(\mathrm{RBS})^{27,28}$ and $\mathrm{x}$-ray photoemission spectroscopy (XPS) measurements it was shown that during annealing the oxygen of the electrodes diffused from the interface into the barrier. ${ }^{29}$ This leads to a larger polarization at the boundary surface between the insulator and electrode and therefore increases the TMR. The difference of the peak value between the forward and backward cycling in the hysteresis loop is considerable. This is caused by the noncomplete alignment of the pinned $\mathrm{Co}_{75} \mathrm{Fe}_{25}$ layer.

Although RBS and XPS are essential for investigating the barrier and interface properties the integral character of these techniques make them rather insensitive to individual (and rare) structural defects. The current transport in tunnel junctions is often determined by interface states and traps. Shang et al. ${ }^{30}$ and more recently Hagler, Kinder, and Bayreuther, ${ }^{31}$ separated the magnetic tunnel junction conductance in three parts. They explained successfully the temperature dependence of magnetic tunnel junctions. The equation

$G=G_{D}(T)+G_{D}(T) \cdot P_{1}(T) \cdot P_{2}(T) \cos (\theta)+G_{I N}(T)$

served for the analysis of the conductivity measurements. Here $G_{D}(T)$ indicates the direct tunneling conductivity and $G_{I N}(T)$ represents the conductance via localized states. Both terms are spin independent. Here it is important to mention that we only collect data in the "high-temperature" regime, that means $e V \ll k T$. If the temperature is $10 \mathrm{~K}$ the thermal energy is around $8 \mathrm{mV}$, whereas our conductance analysis took only data with $|V|<1 \mathrm{mV}$ into account. The term $G_{D}(T) \cdot P_{1}(T) \cdot P_{2}(T) \cos (\theta)$ denotes the spin dependent part wherein the polarization of the bottom and top magnetic layer is indicated with $P_{1}$ and $P_{2}$, respectively, and $\theta$ is the angle between the magnetizations of the top and bottom electrode. As in previous works, ${ }^{30,31}$ we assume the polarization $P$ to be proportional to the magnetization $M$. Therefore we can express the temperature dependence of the polarization according to Bloch's law as

$$
P(T)=P_{0}\left(1-\alpha T^{3 / 2}\right),
$$

in which $\alpha$ indicates a material dependent parameter. Although not absolutely justified by theory, $\alpha$ is often described in literature as the spin wave parameter. ${ }^{30,31}$

For the parallel alignment we have applied a magnetic field of $H=-400 \mathrm{mT}$ (see Fig. 1). For the antiparallel alignment of the magnetization we had to saturate the layer with a positive value of the magnetic field and then apply a field strength of $H_{1}=-9.1 \mathrm{mT}$ to reach the maximum of the TMR curve. $H_{1}$ is indicated in Fig. 1.

For the antiparallel alignment of the electrodes Eq. (1) is given by the expression

$$
G_{\min }(T)=G_{D}(T) \cdot\left[1-P(T)^{2}\right]+G_{I N}(T),
$$

likewise the parallel alignment is described by

$$
G_{\min }(T)=G_{D}(T) \cdot\left[1+P(T)^{2}\right]+G_{I N}(T) .
$$

The term $G_{D}(T)$ is caused by the temperature dependence of the Fermi distribution and leads to the expression ${ }^{32}$

$$
G_{D}(T)=G_{0} \cdot \frac{C T}{\sin (C T)} \quad \text { with } \quad C=1.39 \times 10^{-4} \cdot \frac{d}{\sqrt{\phi}} .
$$

Here $d$ is in $\AA$ and $\phi$ in $\mathrm{eV}$. The constant $C$ depends only on the barrier thickness $d$ and the barrier height $\phi$. For the calculation of $G_{D}(T)$ we insert the value of $d=17.55 \AA$, $\phi_{1}=0.96 \mathrm{eV}$, and $\phi_{2}=0.62 \mathrm{eV}$ which we get from a fit rou- 


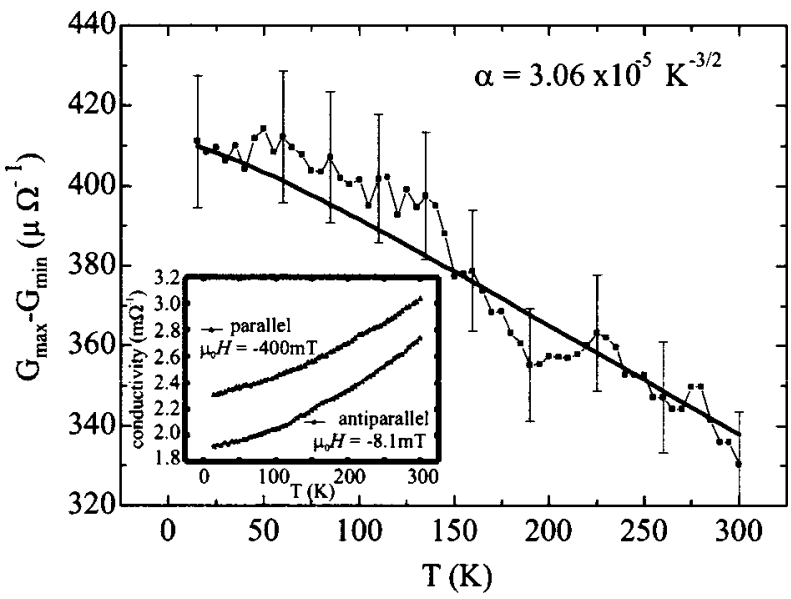

FIG. 2. Temperature dependence of $\Delta G$ annealing. The solid line indicates the fitting with Eq. (8). The inset shows the conductivity in dependence of the temperature for the parallel and antiparallel alignment. The spin wave parameter is determined to $3.45 \times 10^{-5}$ and $2.03 \times 10^{-5} \mathrm{~K}^{-3 / 2}$ before and after annealing, respectively.

tine using the Brinkman formula, ${ }^{35}$ only taking the values for $|V|$ in the voltage interval \pm 20 to $\pm 100 \mathrm{mV}$ into account to avoid the contribution of the zero bias anomaly of magnetic tunnel junctions at low temperatures. This was extracted from an $I-V$ curve measured at $4 \mathrm{~K}$ to minimize the contributions of conductance channels through localized states.

After annealing we can see that the asymmetry of the barrier which we get from the Brinkman fit reduces from $\Delta \phi=-0.34 \mathrm{eV}$ before annealing to $\Delta \phi=+0.12 \mathrm{eV}$ which indicates that the surface contamination will become less due to the annealing procedure.

The inset in Fig. 2 shows the temperature-dependent conductivity for the sample in dependence of the temperature for parallel and antiparallel alignment. To get this curve, we have recorded the conductivity at each temperature at different applied voltages and taken the value of $G$ from the minimum voltage value $(U=0.39 \mathrm{mV})$.

The extrapolation of the conductivity to $T=0 \mathrm{~K}$ yields the following formulas:

$$
\begin{aligned}
& G_{\max }(0)=G_{0} \cdot\left(1+P_{0}^{2}\right), \\
& G_{\min }(0)=G_{0} \cdot\left(1-P_{0}^{2}\right) .
\end{aligned}
$$

The computation of the polarization $P_{0}$ by Eqs. (6) and (7) yields $P_{0}=31.4 \%$. By taking the difference $G_{\max }-G_{\min }$ we can determine the parameter

$$
\begin{aligned}
\Delta G & =G_{\max }-G_{\min }=2 G_{D}(T) P(T)^{2} \\
& =2 G_{0} \frac{C T}{\sin (C T)} \cdot P_{0}^{2} \cdot\left(1-\alpha T^{3 / 2}\right)^{2} .
\end{aligned}
$$

$G_{0}, C$ and $P_{0}$ are known parameters, so we can extract $\alpha$ by fitting the experimental data using Eq. (8).

Due to the low signal-to-noise ratio we can see that the determination of the parameter $\alpha$ is difficult (see Fig. 2). In addition the plateau for the antiparallel alignment is too small (see Fig. 1). If the adjusted magnet field value varies, then one could be immediately on the steep flank of the TMR curve. Nevertheless, all of the measured values lay within an

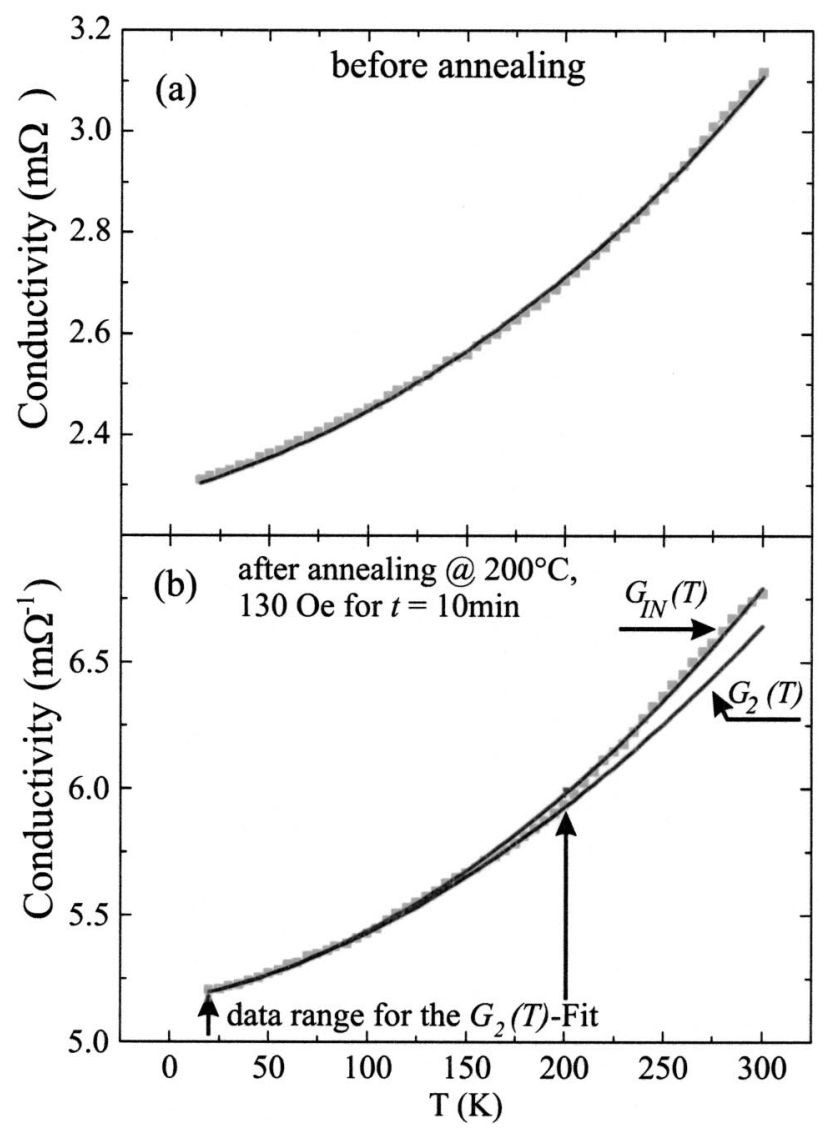

FIG. 3. Conductivity of the as-deposited and annealed sample at parallel alignment. The fitted exponent of the formula of $G_{I N}$ is equal to $n_{2}=1.5$ for the as-deposited sample. The arrows in (b) indicate the range for the $G_{2}$ fit, for which the exponent comes out to $n_{2}=1.32$. The whole data range is used for the $G_{I N}$ fit. The $n_{3}$ exponent is equal to $n_{3}=2.65$.

error of $4 \%$ with regard to the fit curve. The spin wave parameter $\alpha$ was determined to $3.06 \times 10^{-5} \mathrm{~K}^{-3 / 2}$ and to 2.03 $\times 10^{-5} \mathrm{~K}^{-3 / 2}$ before and after annealing, respectively. This result is consistent with the model that a higher surface contamination leads to a larger spin wave parameter $\alpha^{30,33,34}$ This means that the excitation of the spin waves is more difficult than in the case of the as-deposited sample. This suggests a better interface between insulator and electrode after annealing and will be discussed later.

The decrease of the spin polarization between 0 and 300 $\mathrm{K}$ before annealing amounts to $17.9 \%$ when using Eq. (2) with the $\alpha$ obtained here. After annealing the spin polarization decreases by $10.7 \%$ for the same temperature interval. Since we assume spin polarization $P$ and magnetization $M$ to be proportional the large difference between our values and the temperature dependence of the bulk magnetization of $\mathrm{Co}_{75} \mathrm{Fe}_{25}$ has to be discussed. The discrepancy is due to the fact that tunneling is surface sensitive. It is likely that the relevant monolayers at the interface have a considerably different electronic structure compared to bulk and so give rise to different temperature behavior.

A complete description of the temperature dependence $G_{I N}$ remains to be determined. With

$$
G_{\max }=G_{D} \cdot\left[1+P(T)^{2}\right]+G_{I N}
$$


one can fit the spin independent part. In Fig. 3(a) one can see the measured conductance versus temperature substracted by the direct conductance calculated using Eq. (5). If the temperature dependence of the conductance is only due to the direct tunneling the measured conductance in Fig. 3(a) should be a constant. As one can see this is not the case. This means that the tunnel barrier consists of further conductance channels beside the direct tunneling transport. A possible mechanism is tunneling through oxygen vacancies (localized states). This temperature behavior is well-described by the theory of Glazman and Matveev ${ }^{2}$ and has been successfully applied for $\alpha$-Si barriers by the Stanford group. ${ }^{5}$

Besides the direct and resonant tunneling processes, which are temperature independent, the tunneling over $N$ $=2$ localized states has the largest contribution to the temperature dependence of the conductance. If the tunnel process takes place over $N=2$ localized states one can describe ${ }^{2,5}$ this conductivity by $G_{I N}=G_{2} \cdot T^{n_{2}}$ with a theoretical prediction of $n_{2}=1.33$. By fitting the conductivity of the as-deposited sample [Fig. 3(a), solid line], we get a value of $n_{2}=1.5$. This discrepancy with the theoretical value will be explained later.

The same procedure is done with the annealed sample [see Fig. 3(b)]. From $T=15 \mathrm{~K}$ up to $T=200 \mathrm{~K}$ the exponent $n_{2}$ comes out to be $n_{2}=1.32$, in good agreement with the theoretical prediction for $N=2$ localized states. If this function is extrapolated to higher temperatures there is a difference between the experimental data and the theoretical curve. Therefore the next higher exponent $n_{3}=2.5$ has to be taken into account, indicating tunneling via three localized states. If we fit the whole data range with this additional term the exponent will be $n_{3}=2.65$. This is in good agreement with the theoretical prediction for $N=3$ localized states.

The obtained results of this work are consistent with the following qualitative model. In Fig. 4(a) a cross-sectional viewgraph of the as-deposited sample is shown assuming a partially oxidized CoFe bottom electrode as a result of the overoxidized aluminum layer. This forms a thin $(\mathrm{CoFe})_{x} \mathrm{O}_{1-x}$ layer at one interface, as shown in Fig. 4(a), which has at least two effects on the tunnel magnetoresistance junction. On one hand, the interface layer may increase the magnon scattering and therefore reduces the TMR value. On the other hand, it is known that transition metal oxides tend to have a high number of oxygen vacancies. ${ }^{35,36}$ This high number of localized states influences the temperature dependence of the conductance. The discrepancy between the fitted value in Fig. 3(a) is due to the nonuniform accumulation of oxygen vacancies at one interface. Therefore a precondition of the Glazman-Matveev model is not fulfilled. This model assumes an equidistant distribution of the traps in the barrier which is definitely not the case in a $(\mathrm{CoFe})_{x} \mathrm{O}_{1-x}$ interface layer.

Upon annealing, the oxygen diffuses from the $(\mathrm{CoFe})_{x} \mathrm{O}_{1-x}$ layer into the barrier [Fig. 4(b)]. Hence we can assume a uniform distribution of the traps in the barrier and the conductance can be described by the model of GlazmanMatveev. This is confirmed by the good agreement of the exponent for the tunneling over $N=2$ and $N=3$ localized states. Additionally, the interface between the electrode and (a) before annealing

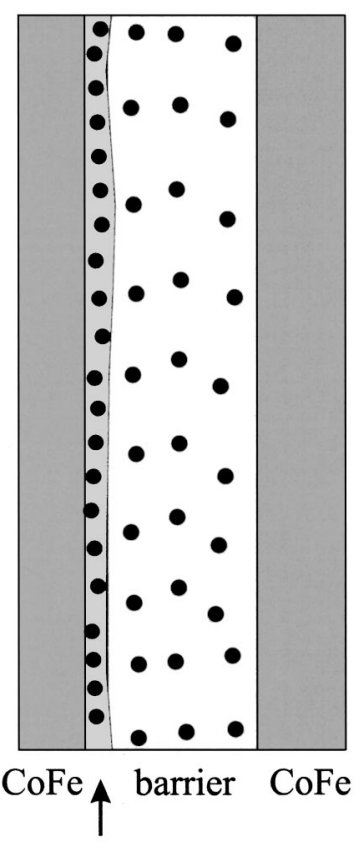

$(\mathrm{CoFe})_{\mathrm{x}} \mathrm{O}_{1-\mathrm{x}}$ (b) after annealing

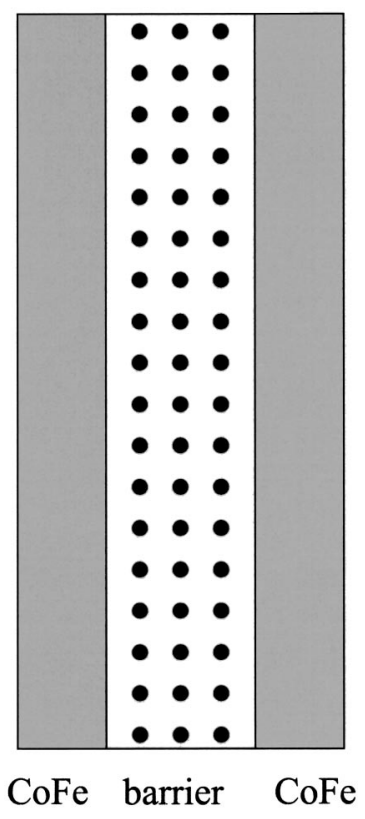

- traps (oxygen vacancies)

\section{layer}

FIG. 4. Illustration of the annealing on a tunnel magnetoresistance junction. In Fig. (a) a thin $(\mathrm{CoFe})_{x} \mathrm{O}_{1-x}$ layer is built probably due to the overoxidation. There are a lot of traps in this layer. Upon annealing the oxygen moves into the barrier and we can assume an uniform distribution of the traps $[(b)]$.

the barrier will be of better quality which is expressed by a lower value of the spin wave parameter $\alpha$.

We would like to point out that the results used in this work are based on a semiempirical approach. For a deeper understanding of the microscopically mechanism a selfconsistent theory as developed by Zhang et al. has to be used. $^{37}$

\section{SUMMARY}

In conclusion, the TMR value increases when the structure is annealed. This is due to the homogenization of the barrier. By measuring the conductivity with and without a magnetic field we are able to separate those into a spinindependent and spin-dependent part. For the annealed sample the spin-independent part reveals that the process can be described by a tunneling over three localized states in the barrier. The oxygen diffusion during annealing results in a smaller spin wave parameter and a reduced number of oxygen vacancies. This is consistent with former RBS and XPS experiments.

\section{ACKNOWLEDGMENTS}

The authors thank D. Bürgler for helpful discussions. This work was supported by the HGF-Strategiefonds "Magnetoelectronics" and the BMBF Leitprojekt "Magnetoelectronics." 
${ }^{1}$ N. F. Mott and E. A. Davis, Electronic Processes in Non- Crystalline Materials, Monographs in Physics (Clarendon, Oxford, 1971).

${ }^{2}$ L. I. Glazman and K. A. Matveev, Sov. Phys. JETP 67, 1276 (1988).

${ }^{3}$ E. L. Wolf, Principles of Tunneling Spectroscopy (Plenum, New York, 1985).

${ }^{4}$ J. Frenkel, Phys. Rev. 36, 1604 (1930).

${ }^{5}$ Y. Xu, D. Ephron, and M. R. Beasley, Phys. Rev. B 52, 2843 (1995).

${ }^{6}$ C. T. Rogers and R. A. Buhrman, Phys. Rev. Lett. 53, 1272 (1984); 55, 859 (1985).

${ }^{7}$ M.J. Kirton and M. J. Uren, Appl. Phys. Lett. 48, 1270 (1986); Adv. Phys. 38, 367 (1989).

${ }^{8}$ M.-H. Tsai, H. Muto, and T. P. Ma, Appl. Phys. Lett. 61, 1691 (1992).

${ }^{9}$ R. T. Wakai and D. J. Van Harlingen, Phys. Rev. Lett. 58, 1687 (1987).

${ }^{10}$ H. Kohlstedt, K.-H. Gundlach, and S. Kuriki, J. Appl. Phys. 73, 2564 (1993).

${ }^{11}$ L. Pearson, R. P. Robertazzi, R. A. Buhrman, S. R. Cypher, and B. D. Hunt, Phys. Rev. Lett. 67, 2866 (1991).

${ }^{12}$ B. Savo, F. C. Wellstood, and J. Clark, Appl. Phys. Lett. 50, 1757 (1987).

${ }^{13}$ M. Naito and M. R. Beasley, Phys. Rev. B 35, 2548 (1987).

${ }^{14}$ S. J. Bending and M. R. Beasley, Phys. Rev. Lett. 55, 324 (1985).

${ }^{15}$ R. Merservey, P. M. Tedrow, and J. S. Brooks, J. Appl. Phys. 53, 1563 (1982).

${ }^{16}$ C. T. Rogers, R. A. Buhrman, H. Kroger, and L. N. Smith, Appl. Phys. Lett. 49, 1107 (1986).

${ }^{17}$ T. Miyazaki and N. Tezuka, J. Magn. Magn. Mater. 139, L231 (1995).

${ }^{18}$ S. S. P. Parkin et al., J. Appl. Phys. 85, 5828 (1999).

${ }^{19}$ H. Boeve, R. J. M. Van de Veerdonk, B. Dutta, J. de Boeck, J. S. Moodera, and G. Borghs, J. Appl. Phys. 83, 6700 (1998).

${ }^{20}$ J. S. Moodera, L. R. Kinder, T.-M. Wong, and R. Merservey, Phys. Rev. Lett. 74, 3273 (1995).
${ }^{21}$ M. Jullière, Phys. Lett. A 54, 225 (1995).

${ }^{22}$ J. S. Moodera and L. R. Kinder, J. Appl. Phys. 79, 4724 (1996); R. Jansen and J. S. Moodera, ibid. 83, 6682 (1998).

${ }^{23}$ J. Zhang and R. H. White, J. Appl. Phys. 83, 6512 (1998).

${ }^{24}$ D. C. Tsui, R. E. Dietz, and L. R. Walker, Phys. Rev. Lett. 27, 1729 (1971).

${ }^{25}$ P. Rottländer, H. Kohlstedt, P. Grünberg, and E. Girgis, J. Appl. Phys. 87, 6067 (2000).

${ }^{26}$ X. F. Han, M. Oogane, H. Kubota, Y. Ando, and T. Miyazaki, Appl. Phys. Lett. 77, 283 (2000).

${ }^{27}$ R. C. Sousa, J. J. Sun, V. Soares, P. P. Freitas, A. Kling, M. F. da Silva, and J. C. Soares, Appl. Phys. Lett. 73, 3288 (1998).

${ }^{28}$ M. F. Gillies, W. Oepts, A. E. T. Kuiper, R. Coehoorn, Y. Tamminga, J. H. M. Snijders, and W. M. A. Bik, IEEE Trans. Magn. 35, 2991 (1999).

${ }^{29}$ S. Therani, J. M. Slaughter, F. Chen, M. Derlam, J. Shi, and M. de Herera, IEEE Trans. Magn. 35, 2814 (1999).

${ }^{30}$ C. H. Shang et al., Phys. Rev. B 58, 2917 (1998).

${ }^{31}$ T. Hagler, R. Kinder, and G. Bayreuther, J. Appl. Phys. 89, 7570 (2001).

${ }^{32}$ R. Stratton, J. Phys. Chem. Solids 23, 1177 (1962).

${ }^{33}$ D. T. Pierce, R. J. Celotta, J. Unguris, and H. C. Siegmann, Phys. Rev. B 26, 2566 (1982); D. Mauri, D. Scholl, H. C. Siegmann, and E. Kray, Phys. Rev. Lett. 61, 758 (1988).

${ }^{34}$ J. Mathon and S. B. Ahmad, Phys. Rev. B 37, 660 (1988).

${ }^{35}$ W. F. Brinkman, R. C. Dynes, and J. M. Rowell, J. Appl. Phys. 41, 1915 (1970).

${ }^{36}$ J. Halbritter, Appl. Phys. A: Mater. Sci. Process. 68, 153 (1999); D. G. Walsmely, E. L. Wolf, and J. W. Osmun, Thin Solid Films 62, 61 (1979).

${ }^{37}$ S. Zhang, P. M. Levy, A. C. Marley, and S. S. P. Parkin, Phys. Rev. Lett. 79, 3744 (1997). 